\title{
JUNO Central Detector and its Calibration System
}

\section{Qingmin Zhang', Yuhang Guo}

Department of Nuclear Science and Technology, School of Energy and Power Engineering, Xi'an Jiaotong University, Xi'an 710049, China

\section{Yuekun Heng, Li Zhou, Boxiang Yu}

Center of Experimental Physics, Institute of High Energy Physics, Beijing 100049, China

\section{Jianglai Liu, Mengjiao Xiao, Feiyang Zhang}

School of Physics, Shanghai Jiaotong University, Shanghai 200240, China

On behalf of JUNO collaboration

\begin{abstract}
The Jiangmen Underground Neutrino Observatory (JUNO) is designed to primarily measure the neutrino Mass Hierarchy. Its Central Detector (CD), which is made of 20 kton Liquid Scintillator (LS), about 18000 20-inch PMTs and 36000 3-inch PMTs, will be the largest LS detector to achieve an unprecedented energy resolution of 3\%@1 @eV and an energy nonlinearity better than $1 \%$. In the meanwhile, an acrylic spherical shell with a diameter of $35.4 \mathrm{~m}$ and a stainless steel-latticed shell with a diameter of $40.1 \mathrm{~m}$ will be constructed for LS container and PMT deployment respectively. Furthermore, a calibration complex is designed for multiple source deployment, the energy coverage of reactor neutrinos and CD full-volume coverage. In this proceeding, the design details and primary progress about the central detector and its calibration system are presented.
\end{abstract}

38th International Conference on High Energy Physics

3-10 August 2016

Chicago, USA

\footnotetext{
${ }^{1}$ Speaker, Corresponding author,E-mail: zhangqingmin@mail.xjtu.edu.cn 


\section{JUNO Introduction}

The Jiangmen Underground Neutrino Observatory (JUNO) is under construction at Kaiping, Jiangmen in southern China and is $53 \mathrm{~km}$ away from both Taishan and Yangjiang Nuclear Power Plant. It is designed to primarily determine the neutrino Mass Hierarchy $(\mathrm{MH})$ by using an underground liquid scintillator detector with an overburden of 700-meter rock for shielding of cosmic rays. JUNO also has other physical potentials. ${ }^{[1]}$

As illustrated in fig.1(a), JUNO is a multiple-purpose neutrino observatory and consists of Central Detector (CD), Calibration System, and Veto Detector. According to the design, the MH will be determined at the level of 3 3.5 sigma during 6-years running ${ }^{[2,3,4]}$. JUNO central detector and its calibration system will be introduced in this proceeding.

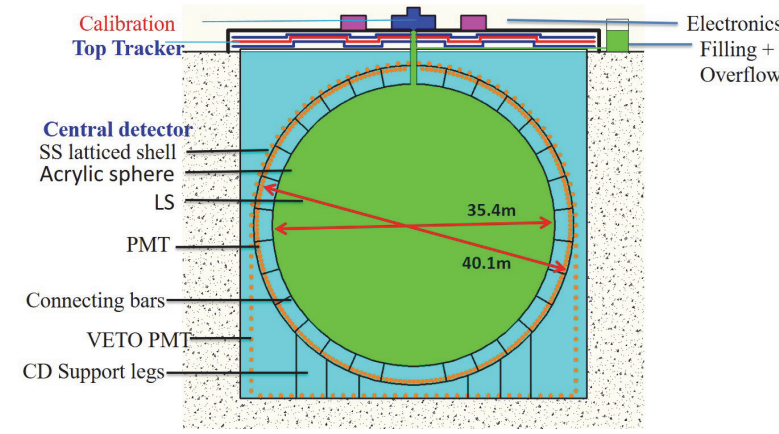

(a)

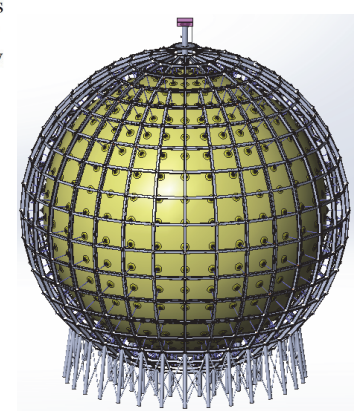

(b)

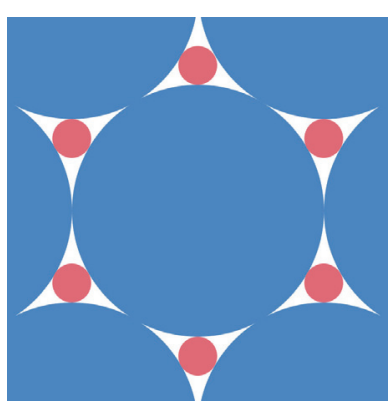

(c)

Figure 1 JUNO detector scheme(a), CD structure (b) and mixture of 20-inch and 3-inch PMTs (c)

\section{JUNO Central Detector}

JUNO CD will be the largest Liquid Scintillator (LS) detector to achieve an unprecedented energy resolution of 3\%@1 MeV. An acrylic spherical shell with a diameter of $35.4 \mathrm{~m}$ and a stainless steellatticed shell with a diameter of $40.1 \mathrm{~m}$ will be constructed for LS container and PMT deployment respectively. The biggest challenges for Liquid Scintillator, PMTs and its structure will be addressed below.

\subsection{Liquid Scintillator}

The LS serves as the target material for reactor anti-neutrino detection. The LS recipe consists of Linear Alkyl Benzene (LAB) as solvent, $3 \mathrm{~g} / \mathrm{L}$ 2,5-diphenyloxazole (PPO) as the fluor and $15 \mathrm{mg} / \mathrm{L} \mathrm{p}$-bis(o-methylstyryl)-benzene (bis-MSB) as the wavelength shifter. ${ }^{[5]}$

Due to its extremely low cross-section, only tens of reactor anti-neutrinos per day can be observed, so it is extremely important to improve the signal-to-noise ratio. Accordingly, the comprehensive light yield is required to be greater than 1200 p.e./MeV and LS's attenuation length should be longer than $20 \mathrm{~m}$ at $430 \mathrm{~nm}$ wavelength, which will be increased by $\mathrm{Al}_{2} \mathrm{O}_{3}$ column purification. Furthermore, the radioactive concentrations of ${ }^{238} \mathrm{U},{ }^{232} \mathrm{Th}$ and ${ }^{40} \mathrm{~K}$ should be below $10^{-15} \mathrm{~g} / \mathrm{g}, 10^{-15} \mathrm{~g} / \mathrm{g}$ and $10^{-17} \mathrm{~g} / \mathrm{g}$ respectively ${ }^{[6,7]}$, and a system with functions of distillation, water extraction and steam stripping will be used to reduce the radiation background. Currently, a purification prototype has been built in Daya Bay onsite.

\subsection{PMTs}

PMTs will be used to detect the scintillation photons from IBD events in LS. About 18,000 20-inch PMTs will be used for CD: about 13,000 MCP-PMTs are supplied by North Night Vision Technology (NNVT) and 5,000 R12860 PMTs are from Hamamatsu. Generally, the PMTs from the two companies are similar in performances, and it's worth mentioning the PMT's detection efficiency (= quantum efficiency $\times$ collection efficiency) is required to be $27 \%$ on average and $24 \%$ on minimum. However, NNVT PMTs have a lower after-pulse percentage and glass radioactivity for lower background, while Hamamatsu PMTs have a shorter transit-time spread for better vertex reconstruction due to its better timing. Additionally, as shown in Fig.1 (c), 36,000 3-inch PMTs are also deployed to serve as an additional standalone calorimetry because of no saturation and better linearity in JUNO situation. ${ }^{[8]}$

Some challenges, such as waterproof potting, mechanical PMT protection ${ }^{[9]}$, geomagnetism 
shielding, are in our consideration and have had baseline solutions.

\subsection{Structure}

The CD structure is composed of an acrylic shell with $35.4 \mathrm{~m}$ in diameter for LS container and a stainless steel-latticed shell with $40.1 \mathrm{~m}$ in diameter for PMT deployment. 590 connecting bars are deployed between them for stable connection. The 3-D design visualization is displayed in fig.1(b).

The acrylic shell with 21 layers is made of 291 pieces of acrylic bricks with a thickness of $120 \mathrm{~mm}$. Top chimney and bottom flange are designed for LS filling. Due to pure water outside the acrylic shell, LS and water filling will take place simultaneously considering the difference of their densities. Meanwhile, owing to LS volume change from temperature change, overflow tank and temperature controls have been considered. During filling, the LS will be sealed with nitrogen and the liquid level, flow and acrylic stress will be monitored. FEA shows maximum stress of acrylic is below the required $3.5 \mathrm{Mpa}$ when tensile load is less than 8.2 ton. ${ }^{[10]}$

\section{Calibration Systems and Preliminary Simulation}

In order to achieve an overall energy resolution of $3 \%$ at $1 \mathrm{MeV}$ and an energy nonlinearity less than $1 \%$, a complete calibration complex is designed for multiple source deployment, the entire energy coverage of reactor neutrinos and $\mathrm{CD}$ full-volume coverage.

\subsection{Calibration Systems}

As demonstrated in Fig.2 (a), the calibration complex includes Automatic Calibration Unit (ACU), Cable Loop System (CLS), Guide Tube Control System(GTCS) and Remotely Operated under-liquidscintillator Vehicles (ROV), whose key features are listed table 1.

Table 1 key features of calibration sub-systems

\begin{tabular}{|c|c|c|c|c|c|}
\hline Demision & Sub-ystem & Calibration Region & $\begin{array}{l}\text { Position } \\
\text { Control }\end{array}$ & Positioning & Purpose \\
\hline $1-\mathrm{D}$ & $\mathrm{ACU}$ & along central axis & \multirow{3}{*}{$\begin{array}{c}\text { Spool drive } \\
\text { ( Rope is steel } \\
\text { wire coated with } \\
\text { Teflon) }\end{array}$} & Rope Length Calculation & These three \\
\hline \multirow[b]{2}{*}{$2-\mathrm{D}$} & CLS & $\begin{array}{l}\text { in one given vertical } \\
\text { plane }\end{array}$ & & $\begin{array}{l}\text { Rope Length Calculation, } \\
\text { Ultrasonic Receiver, CCD }\end{array}$ & $\begin{array}{l}\text { are very } \\
\text { critical and }\end{array}$ \\
\hline & GTCS & $\begin{array}{c}\text { along a given } \\
\text { longitude of acrylic } \\
\text { sphere }\end{array}$ & & Rope Length Calculation & $\begin{array}{l}\text { combined for } \\
\text { routine } \\
\text { calibration }\end{array}$ \\
\hline $3-\mathrm{D}$ & ROV & $\begin{array}{c}\text { whole of the CD } \\
\text { volume }\end{array}$ & $\begin{array}{c}\text { Remotely } \\
\text { Operated Vehicle }\end{array}$ & Ultrasonic Receiver, CCD & Insurance \\
\hline
\end{tabular}

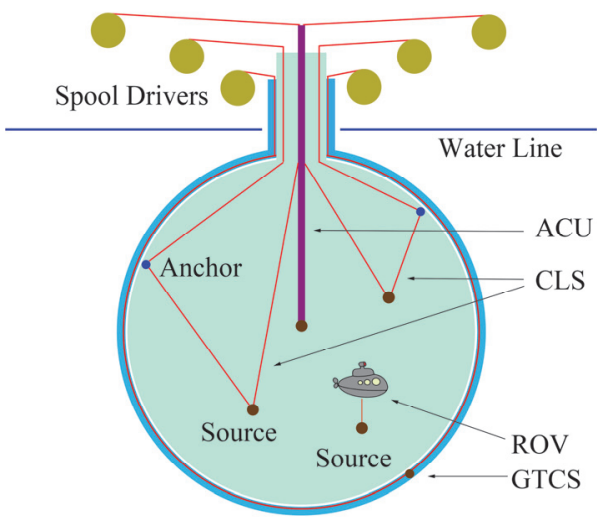

(a)

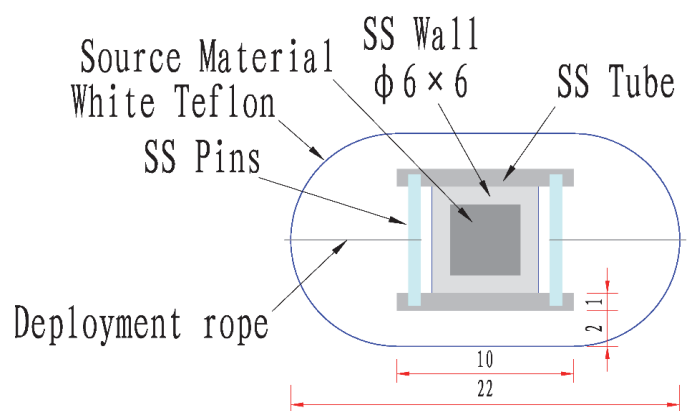

(b)

Figure 2 Schemetic diagram of calibration complex and the source enclosure design (not to scale)

The radiation sources include neutron sources $\left({ }^{241} \mathrm{Am}-\mathrm{Be},{ }^{241} \mathrm{Am}-{ }^{13} \mathrm{C}\right.$ or ${ }^{241} \mathrm{Pu}-{ }^{13} \mathrm{C},{ }^{252} \mathrm{Cf}$ ), position sources $\left({ }^{22} \mathrm{Na},{ }^{68} \mathrm{Ge}\right)$ and gamma sources $\left({ }^{40} \mathrm{~K},{ }^{54} \mathrm{Mn},{ }^{60} \mathrm{Co},{ }^{137} \mathrm{Cs}\right)$. The source enclosure has been designed for sealing, as shown in Fig. 2 (b) and simulation shows the energy bias due to the enclosure is less than $0.2 \% \cdot{ }^{[11]}$ 


\subsection{Investigation of position-dependent response effect}

For such a big detector, its response strongly depends on the position ${ }^{[11]}$ and the structure effect is not negligible for the boundary volume ${ }^{[12]}$, as shown in Fig. 4 from $1.0 \mathrm{MeV}$ gamma simulation. Full absorption peak is used for the energy measurement. However, the full absorption spectrum overlaps with Compton edge near boundary, so spectrum is fitted with a sum function of Gaussian and Compton tail to obtain the full absorption peak more accurately.

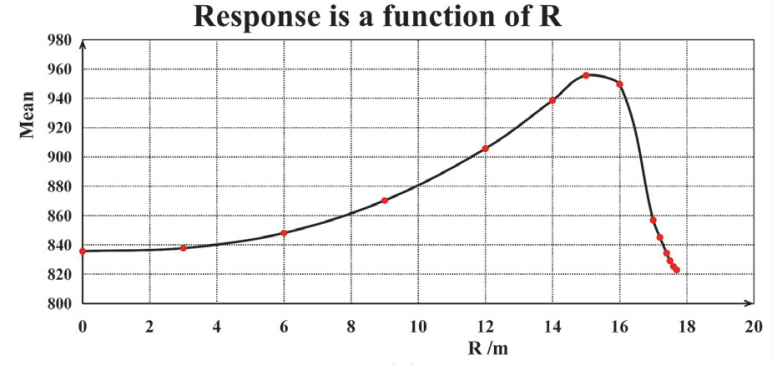

(a)

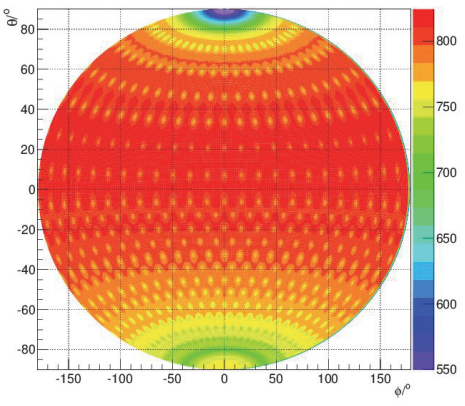

(b)

Figure 3 Energy response's dependence on radius (a) and energy response on the CD surface (b)

Based on the main strategies mentioned above, radius, surface latitude and longitude scan have been carried out. As shown in fig.3 (a), the response's dependence on radius is very obvious, especially near boundary. The non-uniform and periodical response on CD surface indicated in fig. 3 (b) is attributed to CD chimney, copper fasteners and the distribution of PMTs. The CLS and GTCS will be placed in a given longitude, in which the response can represent the average effect. ${ }^{[12]}$

\subsection{Preliminary Correction with ACU,CLS and GTCS Combined}

Since the three calibration systems (ACU, CLS and GTCS) will be combined for routine calibration, the response map for $1.0 \mathrm{MeV}$ gammas has been depicted by simulation on the given points from ACU, CLS and GTCS. Then, the ratio of $(\mathrm{R}, \theta)$ to center has been obtained by fitting with "spline function" and has been used to correct the response of $1.0 \mathrm{MeV}$ gamma events, which are uniform in the CD. Finally, the corrected energy spectrum has been fitted with a Gaussian function, achieving a bias of $0.08 \%$ and a energy resolution 3.29\%@1.0MeV. ${ }^{[12,13]}$

Of course, further improvements and more work are needed and undergoing.

\section{References}

[1] Cao J. Daya Bay and Jiangmen Underground Neutrino Observatory (JUNO) neutrino experiments (in Chinese)[J]. Sci Sin-Phys Mech Astron, 2014, 44:1025-1040, doi: 10.1360/SSPMA2014-00174

[2] L. Zhan, Y.F. Wang, Determination of the neutrino mass hierarchy at an intermediate baseline[J], Phys. Rev. D 78, 111103(R) (2008)

[3] L. Zhan, Y.F. Wang, Experimental requirements to determine the neutrino mass hierarchy using reactor neutrinos[J], Phys. Rev. D 79, 073007 (2009)

[4] Y.F. Li, J. Cao, Unambiguous determination of the neutrino mass hierarchy using reactor neutrinos [J], Phys. Rev. D 88, 013008 (2013)

[5] H. Enzmann, Online attenuation length monitoring during filling[R], Beijing: IHEP, 2016

[6] Wanda Beriguete, et al. Production of a gadolinium-loaded liquid scintillator for the Daya Bay reactor neutrino experiment, Nuclear Instruments and Methods in Physics Research Section A, Volume

763, 1 November 2014, Pages 82-88

[7] J. Zhao, Low background studies[R], Beijing: IHEP, 2016

[8] W. WANG, Plans for Mass PMT Testing of JUNO[R], Beijing: IHEP, 2016

[9] M. He, Status of PMT protection [R], Beijing: IHEP, 2016

[10] X.Y. Ma, Installation of the Central Detector[R], Beijing: IHEP, 2016

[11] J.L. Liu, JUNO Calibration Status[R], Beijing: IHEP, 2016

[12] Q.M. Zhang, Preliminary Design Review: Guide Tube Calibration System for JUNO[R], Beijing:

IHEP, 2016

[13] F.Y. Zhang, CD uniformity calibration strategy[R], Beijing: IHEP, 2016 